\title{
PLACENTAL IGF1R-PI3K/AKT PATHWAY AND ITS RELATIONSHIP TO IDIOPATHIC BIRTH WEIGHT ALTERATIONS
}

\section{Universidad Guanajuato}

Maria-Luisa Lazo-de-la-Vega-Monroy, PhD1, Martha I. GonzálezDomínguez, PhD1, Leonel Daza-Benítez, PhD², Gloria BarbosaSabanero, $\mathrm{PhD}^{1}$.

Medical Sciences Department, Health Sciences Division, University of Guanajuato Leon Campus. 2 UMAE No. 48 IMSS, Leon, Guanajuato. Mexico.

\section{INTRODUCTION}

Alterations in fetal growth lead to neonatal health risks and favor metabolic diseases during adult life (1). Therefore, the study of birth weight determination and its modifications is crucial for metabolic diseases prevention. Placenta growth is a key factor in fetal growth (2). It has been suggested that insulin and the IGF (Insulin-like growth factor) system play an important role in fetal growth and placental development and function (3). Although insulin and IGFs in the umbilical cord blood have been associated to birth weight (4), the roles of placental IGF1 and Insulin receptors (IGF1R and IR), and the PI3K/Akt signaling pathway, shared by both receptors, are not fully elucidated.

\section{OBJECTIVE}

We aimed to analyze protein expression of insulin/IGF receptors and activation of the PI3K/Akt pathway in relation to placental weight and birth weight.

\section{METHODS}

Transversal comparative study in placentas from healthy mothers of term newborns SGA, AGA and LGA (small, adequate and large for gestational age) ( $n=20$ per group). Protein expression of IR, IGF1R and phosphorylation of signaling molecules were analyzed by Western Blot.

\section{RESULTS}

IGF1R expression decreased $20 \%$ significantly in SGA compared to control AGA, and positively correlated with placental weight $(r=0.34, p=0.007)$ and birth weight $(r=0.285$, $p=0.027$ ). IR protein expression was not modified between groups. Phosphorylation of PDK1, main kinase for Akt activation, decreased $40 \%$ in both SGA and LGA compared to control. In line with this, we observed a nearly $30 \%$ decrease in total Akt in SGA and GA compared to AGA. PDK1-dependent phosphorylation pAkt-Thr308 showed tendency, albeit not significant, to decrease in SGA and LGA, while pAkt-Ser273 did not differ between groups.

\begin{tabular}{|c|c|c|c|c|c|}
\hline & $\begin{array}{c}\text { SGA mothers } \\
(\mathrm{n}=\mathbf{2 0})\end{array}$ & $\begin{array}{c}\text { AGA mothers } \\
(\mathbf{n}=\mathbf{2 0 )}\end{array}$ & $\begin{array}{c}\text { LGA } \\
\text { mothers } \\
(\mathbf{n}=\mathbf{2 0})\end{array}$ & $\mathbf{F} / \mathbf{H} \#$ & $\mathrm{p}$ \\
\hline Age (years) & $24.5 \pm 4.7$ & $24.5 \pm 5.6$ & $26.9 \pm 4.7$ & 1.5 & 0.2322 \\
\hline Pregestational Weight $(\mathrm{Kg})$ & $54.9 \pm 10.6$ & $57.1 \pm 9.3 ¥$ & $75.9 \pm 20.8^{*+}$ & 11.7 & $<0.0001$ \\
\hline Height (m) & $1.5 \pm 0.07$ & $1.5 \pm 0.04$ & $1.6 \pm 0.06 \mathrm{Q}^{*+}$ & 2.9 & 0.0612 \\
\hline Pregestational BMI $\left(\mathrm{Kg} / \mathrm{m}^{2}\right)$ & $23.4 \pm 5.3 ¥$ & $23.9 \pm 3.9 \times$ & $30.0 \pm 9.0 d^{*+}$ & 5.9 & 0.0051 \\
\hline Gestational weight gain $(\mathrm{kg}$ ) & $12(10-13)$ & $12(10-18)$ & $12(10-15)$ & 2.1 \# & 0.3495 \\
\hline
\end{tabular}

$\mathrm{n}=19 ; ¥, \mathrm{n}=18 ; \S, \mathrm{n}=16 ; \dot{\phi}, \mathrm{n}=17$. " $\mathrm{p}<0.05$ compared to control AGA. ${ }^{+} \mathrm{p}<0.05$ compared to $\mathrm{SG}, \mathrm{A}$

Table 2. Clinical and anthropometric characteristics of SGA, AGA and LGA newborns.

\begin{tabular}{|c|c|c|c|c|c|}
\hline & $\begin{array}{c}\text { SGA } \\
(\mathbf{n}=\mathbf{2 0})\end{array}$ & $\begin{array}{c}\text { AGA } \\
(\mathbf{n}=\mathbf{2 0})\end{array}$ & $\begin{array}{c}\text { LGA } \\
(\mathrm{n}=20)\end{array}$ & $\mathbf{F} / \mathbf{H} \#$ & $p$-value \\
\hline $\begin{array}{l}\text { Baby's gender } \\
\text { (Male/Female) }\end{array}$ & $9 / 11$ & $12 / 8$ & $12 / 8$ & ND & ND \\
\hline $\begin{array}{l}\text { Delivery method } \\
\text { (Vaginal/C-section) }\end{array}$ & $9 / 11$ & $13 / 7$ & $8 / 12$ & ND & $\mathrm{ND}$ \\
\hline Gestacional age (wk) & $38.2 \pm 1.1$ & $38.7 \pm 1.1$ & $39.4 \pm 0.9^{+}$ & 6.1 & 0.0065 \\
\hline Placental weight (g) & $400.4 \pm 64.3$ & $610.4 \pm 23.8$ & $750.6 \pm 118.7^{*^{+}}$ & 60.6 & $<0.0001$ \\
\hline Birthweight (g) & $\begin{array}{l}2297 \\
(2089-2403)^{*}\end{array}$ & $\begin{array}{l}3273 \\
(2915-3358)\end{array}$ & $\begin{array}{l}3915 \\
(3815-4167)^{*+}\end{array}$ & $52.5 \#$ & $<0.0001$ \\
\hline Birth lenght $(\mathrm{cm})$ & $45.7 \pm 2.5^{*}$ & $50.9 \pm 2.0$ & $52.7 \pm 2.1^{\mathrm{k}^{+}}$ & 53.6 & $<0.0001$ \\
\hline $\begin{array}{l}\text { Cephalic perimeter } \\
\text { (cm) }\end{array}$ & $32.225 \pm 1.6^{*}$ & $34.7 \pm 1.6$ & $36.5 \pm 1.3^{*^{+}}$ & 39.6 & $<0.0001$ \\
\hline $\begin{array}{l}\text { Thoracic perimeter } \\
(\mathrm{cm})\end{array}$ & $29.5(28-31) *$ & $34(32-34)$ & $35(35-37) *^{*^{+}}$ & $45.4 \#$ & $<0.0001$ \\
\hline $\begin{array}{l}\text { Abdominal perimeter } \\
(\mathrm{cm})\end{array}$ & $27.3 \pm 2.0^{*}$ & $30.8 \pm 1.6$ & $34.1 \pm 2.2^{*}$ & 60.2 & $<0.0001$ \\
\hline
\end{tabular}

Mean \pm SD: median (quartile range): F. F value for ANOVA: H. H value of Chi-squared for Kruskal-Walli N.D., not determined $\circ \mathrm{n}=19$. " $\mathrm{p}<0.05$ compared to control $\mathrm{AGA}$. Mean $\mathrm{SD}$; median (quartile range): $\mathrm{F}$, $\mathrm{F}$ value for ANOVA; $\mathrm{H}, a \mathrm{n}=19 .{ }^{*} \mathrm{p}<0.05$ compared to control AGA. ${ }^{+} \mathrm{p}<0.05$ compared to SGA.
Figure 1. IGF1R protein expression (A, B) and its correlation with placental (C) and birth weight (D).
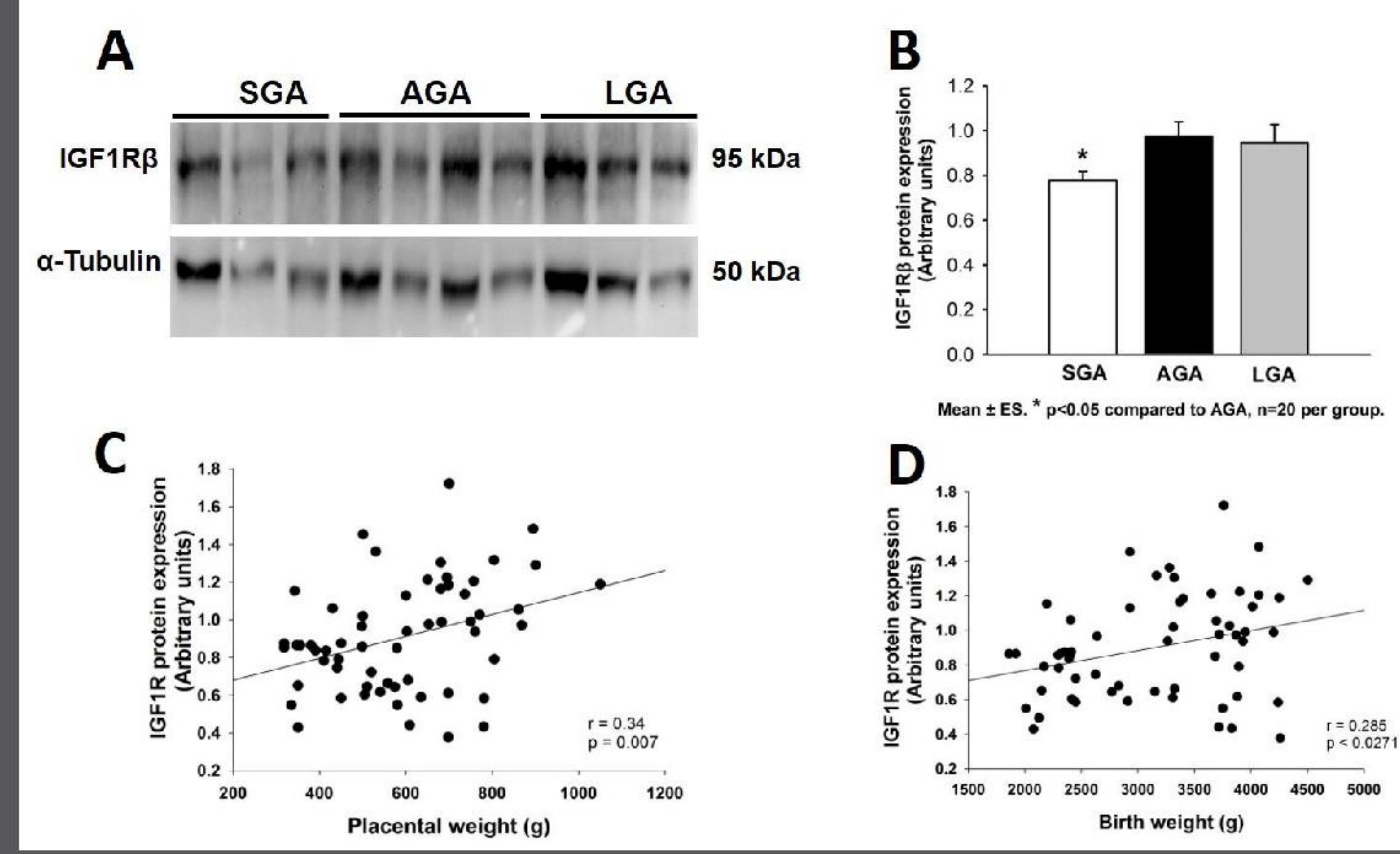

Figure 2. Protein expression and activation of the PI3K/Akt pathway. PDK1 phosphorilation (A), total Akt (B) Akt-Thr308 phosphorylation (C) and AktSer473 phosphorylation (D). Mean \pm ES. ${ }^{*} p<0.05$ compared to $A G A, n=20$ per group.

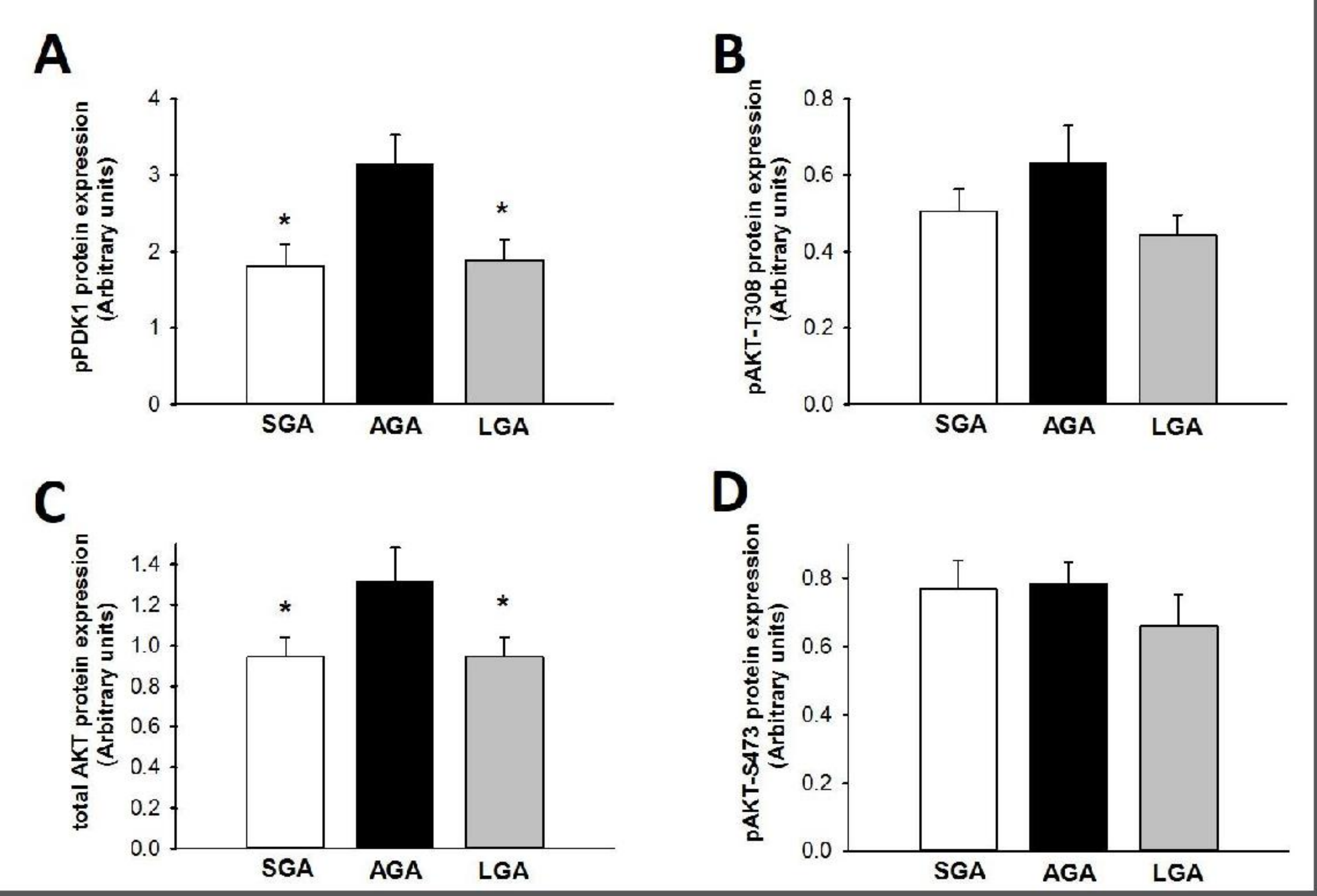

Figure 3. Insulin receptor (INSR) protein expression. Mean \pm ES. $n=20$ per group.

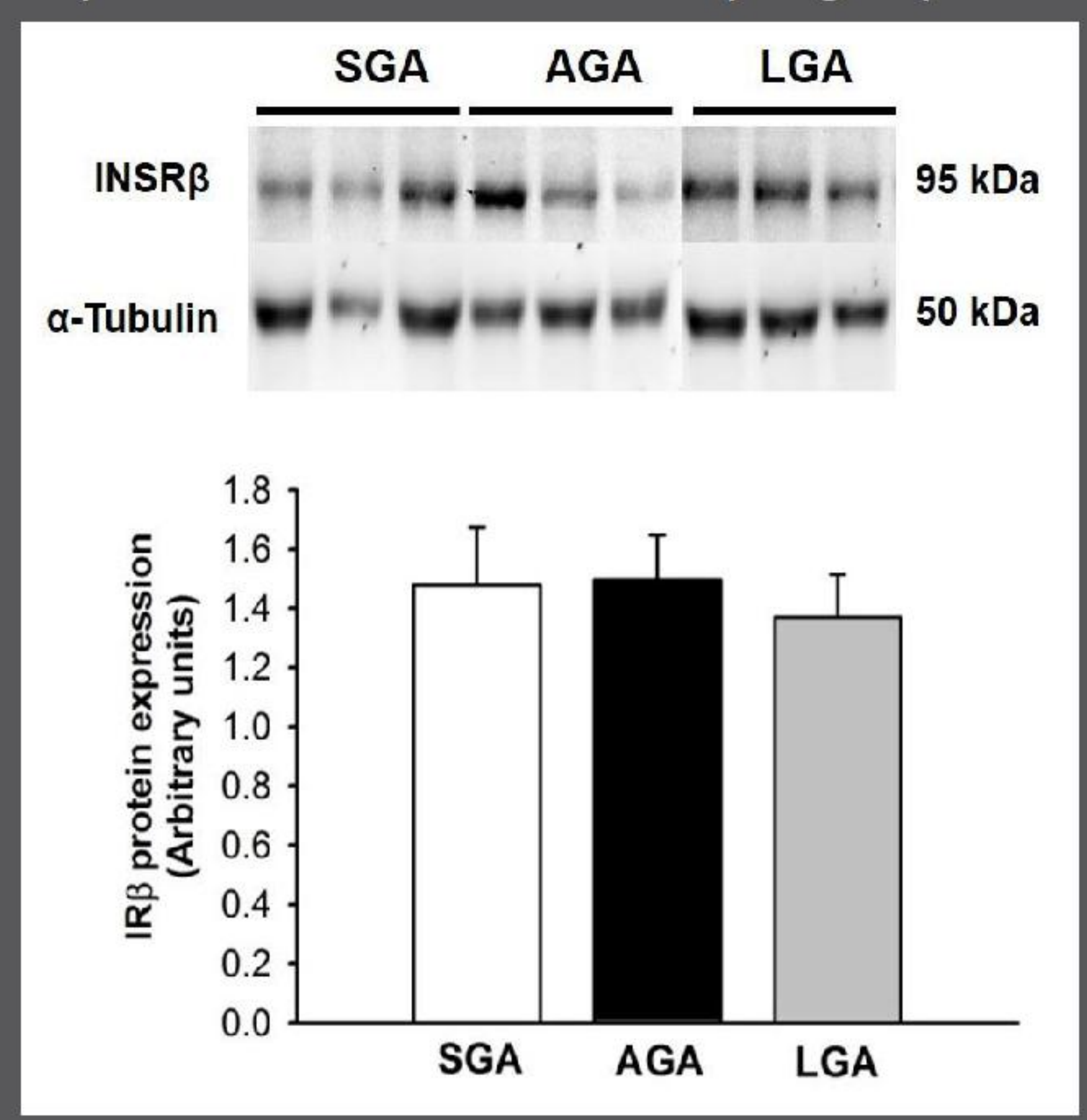

\section{CONCLUSIONS}

These results suggest PI3K/Akt pathway may be differentially regulated in SGA and LGA placentas, possibly related to decreased IGF1R expression in SGA, and perhaps other signaling pathways in LGA, consequently leading to alterations in birth weight. 